\title{
Nanotechnology and Nucleic Acids
}

\author{
Yuri M. Yevdokimov" and V.V. Sytchev
}

Engelhardt Institute of Molecular Biology of the Russian Acad. Sci., 119991 Moscow, Vavilova str. 32, Russia

\begin{abstract}
The peculiarities of two approaches in the nanodesign based on the double-stranded nucleic acid molecules are considered. Main attention is devoted to the formation and properties of nanoconstructions based on the double-stranded DNA molecules and the complexes with chitosan, fixed in the spatial structure of particles of their cholesteric liquidcrystalline dispersions, and cross-linked by artificial nanobridges consisting of alternating copper ions and daunomycin molecules. In contrast to initial DNA liquid-crystalline particles, the structure of resulting nanoconstruction is not "liquidcrystalline" any more; rather it is rigid, crystal-like, three-dimensional structure. The formed nanoconstructions possess the unique spatial and optical properties; this opens a gate for their application in biotechnology and medicine.
\end{abstract}

Keywords: Nanotechnology, nanostucture, biological macromolecules, nucleic acids, chitosan, liquid-crystalline dispersions, nanobridges, circular dichroism, chitosan.

\section{INTRODUCTION: BIOLOGICAL MOLECULES AND NUCLEIC ACIDS AS OBJECTS FOR NANO- TECHNOLOGY}

At the turn of the $21^{\text {st }}$ century, it became clear that individual atoms (or a single molecules) could be used as building blocks for the target-oriented design of spatial structures using the "atom-by-atom" approach. Structures that consist of a limited number of building blocks appear to manifest fundamentally new dominant physical phenomena - quantum effects, statistically temporal variations of properties and their scaling depending on the size of the structures themselves, prevailing influence of surface behavior, absence of defects in the volume, considerable "power intensity" that determines the high chemical reactivity of structures designed, and so on. These novel phenomena are responsible for unique mechanical, electrical, magnetic, optical, chemical, and other characteristics that are opening vistas for manipulating these structures in modes unimaginable under normal conditions. Hence, manipulations at the level of individual atoms have made it possible to design new "structured" materials and devices that possess unique preset properties.

Basic research of phenomena that occur in structures of sizes from $10^{-9}$ to $10^{-12} \mathrm{~m}$ underlies a new field of science and technology known as "nanotechnology". Nanotechnology may be defined as the interdisciplinary science of design and the use of "structured" materials, devices, and systems whose functions are dependent on the nanostructure's geometry or inherent to them specific properties [1].

The materials of recent seminars and symposia held in various countries (see, for instance, NanoTech 2005, May 812, Anaheim, California, USA; NATO-workshop "Nanomaterials for application in medicine and biology", 2006, October 4-6, Bonn, Germany) are indicative of the rapidly expanding nanotechnological studies based on using biological

*Address correspondence to this author at the Institute of Molecular Biology of the RAS, 119991 Moscow, Vavilova str. 32, Russia;

E-mail: yevdokim@eimb.ru macromolecules. This interest in biological molecules is quite justified. In the process of evolution, these molecules acquired properties that are extremely attractive for use in nanotechnology. First, we should emphasize that the chemical diversity of such building blocks as amino acids, lipids, and nucleotides (nucleosides) cannot be compared with that of inorganic ones. Second, biological building blocks tend to a spontaneous - and yet manageable at the molecular level formation of sophisticated spatial structures. Third, there are many approaches for assembling (polymerizing) biological building blocks. The hierarchy of self-assembling biological structures starts with monomers (nucleotides and nucleosides, amino acids, lipids, and so on); followed by biopolymers (DNAs, RNAs, proteins, and polysaccharides); their ensembles (membranes and organelles); and, finally, cells, organs, and even organisms. Fourth, the nanobiomaterials that infrequently result from self-assembling may have both improved characteristics and unique applications. This affords a good opportunity for designing a huge series of nanostructures. Therefore, nanobiotechnology may be defined as a science of creating nanoconstructions (nanobiomaterials) with unique properties, based on biomolecules (their complexes and ensembles). (One could say that biology is a science in which nanobiotechnology really "works").

The combination of the chemical reactivity of biopolymers and their tendency to form hierarchic nanostructures, as well as the opportunity to commercialize biopolymers, makes biological molecules suitable for application in nanotechnology. Therefore, their use in creating artifical nanostructures based on principles furnished by nature appears to be quite logical. Moreover, the progress in chemical synthesis and biotechnology, which allows one to combine building blocks of a variable origin, that is, to design "chimeric" molecules, containing, for instance, amino acids and synthetic organic chains, opens up fantastic vistas for designing nanomaterials and nanostructures that in principle do not exist in nature. It is anticipated that as nanobiotechnology advances, biopolymers will make a transition from the world of biology to that of technology. 
Nanodesign based on the double-stranded (ds) nucleic acids (NA's), i.e. directed creation of three-dimensional (3D), spatial constructions with the tailored properties, "building blocks" of which are ds NA molecules or their complexes with biologically active compounds [2], is a topic of current theoretical and experimental interest [3-13].

The very possibility of using ds NA for formation of nanoconstructions ( $\mathrm{NaC}$ 's) with controlled parameters is based on a few properties characteristic of NA molecules only [2]:

(i) samples of NA molecules of preset sequence of nitrogen bases ranging in length from just a few nucleotides to chains several tens of micrometers long can be obtained in production quantities using routine biotechnological procedures. (This is not the case for any other polymer);

(ii) short helical molecules of ds NA with lengths of the order of $100-1000 \AA$ have a high local rigidity under standard solvent conditions that allows such molecules to be used as "building blocks" without disturbing their physical properties;

(iii) the predicted spatial families of rigid ds NA molecules and the mode of intermolecular interaction under various conditions as well as the intrinsic susceptibility of the NA secondary structure to external stimuli mediated by small molecules or ions, open up a gate for the directed regulation of the characteristics of designed spatial constructions, i.e. to fabricate nanomechanical devices;

(iv) the nitrogen bases in the spatial NA constructions retain their capacity to interact with diverse chemical compounds and biologically active substances, as well as to orient these compounds in respect to the NA molecular axis, which imparts an additional chemical reactivity to the whole structure;

(v) the flexible single-stranded NA "recognizes" its complementary strand and, owing to H-bonds, forms a stable complex with the latter, thus providing an opportunity to prepare a rigid ds molecule with preset composition;

(vi) the formation of branching points in the ds NA molecules combined with complementary (recognizing, "sticky") ends allows one to design plane lattices and sophisticated spatial structures.

Researchers nave become progressively more and more interested in ds NA's, which serve as building blocks for 3-D nanoconstructions with controlled properties. There are two nanodesign strategies, which utilize different NA properties, however, irrespective of the concrete strategy, the structure of nanomaterials based on a ds NA may be controlled to a molecular-level precision.

In this review we have attempted to consider briefly the backgrounds of two different strategies of nanodesign based on the double-stranded DNA molecules, compare the peculiarities of the formed DNA nanoconstructions, and outline the opportunities for the practical application of these nanoconstructions.

\section{NANOCONSTRUCTION DESIGN STRATEGIES}

\subsection{Step-by-Step Design}

Currently, several strategies (approaches) in the design of NA NaC's have been described, which can arbitrarily be referred to as successive or step-by-step design. The first approach is based on successive modification of the initial ds NA (or a synthetic polynucleotide) molecule, and it was pioneered by N. Seeman in 1982 [14] (Fig. 1).

The strarting step [15] in N. Seeman approach consists in preparing NA's fragments with single-stranded "sticky" ends using biochemical procedures or direct chemical synthesis. The interaction between the sticky ends of different NA fragments leads to the formation of a single structure with two breaks in the sugar-phosphate chains (Fig. (1), II). These breaks are cross-linked by enzyme - ligase, and, as a result, a rigid ds NA is formed. The second step is the formation of a branching point (cruciform structure, III) using NA fragments with a specific sequence of nitrogen bases. The cruciform structures may consist of three or four branches; i.e., they can differ in their spatial shapes. Sticky ends are formed in the cruciform ds NA molecule using enzymes. Crosslinking of the sticky ends of these initial molecules results in the formation of the first nanostructure - a plane 2-Dnanolattice composed of ds NA molecules.

Due to mobility of the DNA structure at the branching point, the resulting flate nanolattices is not very rigid. To obtain more rigid nanolattices, a few additional approaches, which may be called "supplementary" to the approach suggested by Seeman have been used. In particular, one of such technique (by D. Bergstrom [16]) is based on the use of molecule composed of two single-stranded selfcomplementary oligonucleotides, the ends of which are bound to each other via a rigid chain of two p-(2hydroxyethyl)-phenylethynylphenyl spacers linked to a tetrahedral carbon atom. The complementary hybridization of oligonucleotide fragments, which results in the formation of ds structure, is accompanied by the development of a set of multiarm-star-shaped nanolattiles. The structural rigidity of this nanolattice is enabled by the sequential alternation of hydrocarbon moieties with a fixed spatial structure and ds oligonucleotides. Another technique by Niemeyer et al. [17] is based on the use of bis(biotinylated) DNA molecule and biotin-binding protein - streptavidin. This allows plane nanolattice shaped as closed rings to be made using reactions of complex formation.

Using an appropriate nucleotide sequence in starting single-stranded DNA's and adequate cruciform structures, N. Seeman obtained a cube-shaped nanoconstruction, in which ds DNA molecules served as the stiffening ribs. Various aspects of step-by-step strategy have been reviewed, different plane lattices, as well as a cube-shaped nanostructure, octahedrons, dodecahedrons, and so on, in which ds DNA molecules served as the ribs, have been discussed [18 ].

It is noteworthy that the design of nanostructures using the technique described above requires large expenditures. Indeed, it is necessary to obtain the NA fragments with specified sequences of nitrogen bases or their complexes with concrete "linkers"; to use a wide set of enzymes (restrictases and ligases) for splitting and cross-linking of NA fragments between desired nitrogen bases, to select specific 


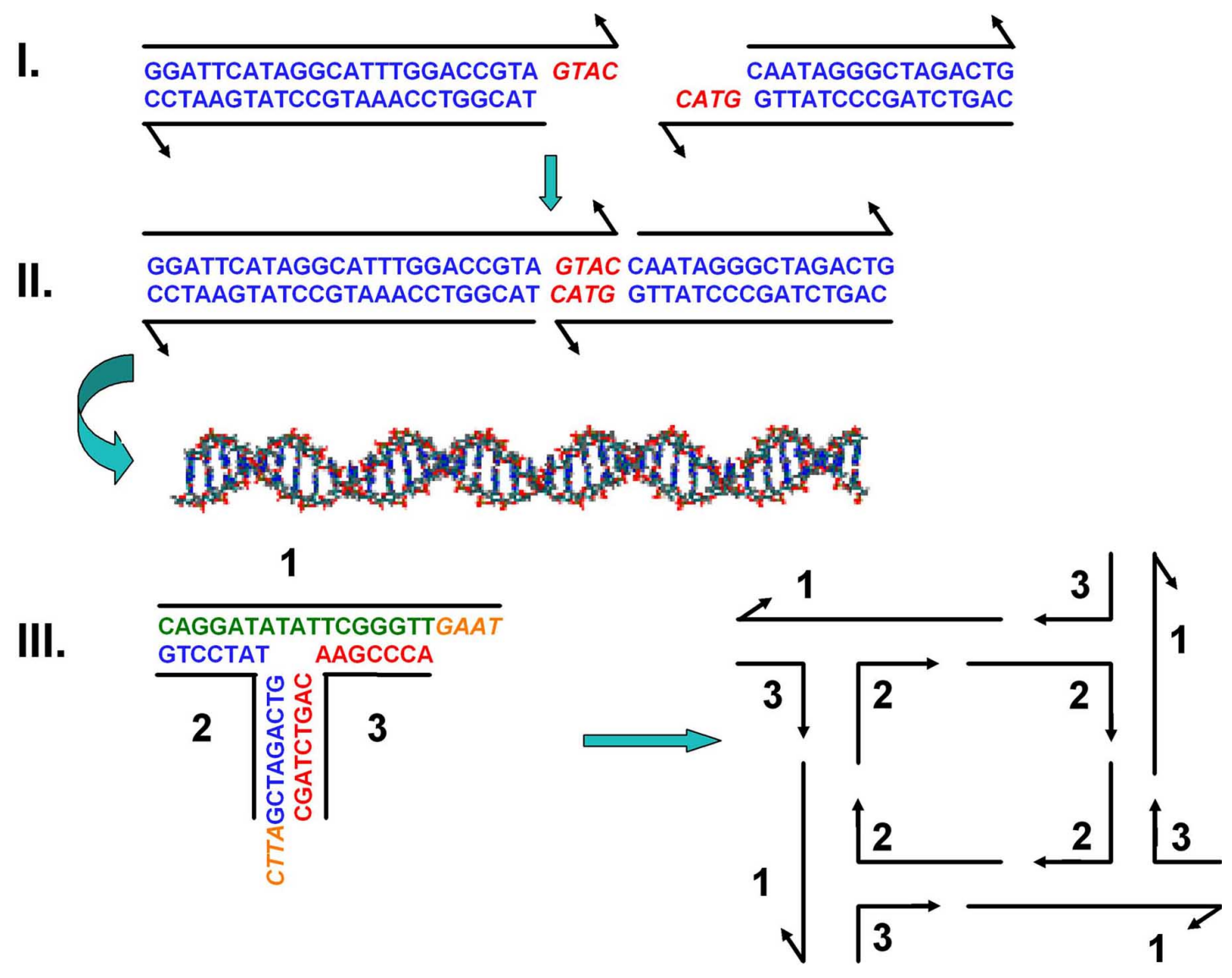

Fig. (1). Formation of a plane lattice from double-stranded nucleic acid molecules. (I) - two initial fragments with sticky ends (the arrows at the ends indicate the 5 ' $\rightarrow 3$ ' direction of sugar-phosphate chains), (II) - valuable ds nucleic acid structure obtained after cross-linking of breaks, and (III) - the cruciform structure and a plane lattice formed from this structure.

structures from the reaction mixture, to perform careful analysis of their properties; and apply modern monitoring methods (such as atomic force microscopy) in all stages of the nanodesign.

One can stress, that the creation of 3-D nanostructures from plane nanolattices does not influence the physical and chemical characteristics of DNA molecules and these molecules retain the parameters of the secondary structure of Bform. This opens a way for introducing molecules of various compounds ("guest" molecules) into content of forming $\mathrm{NaC}$.

The formation of 3-D nanoconstructions with controlled properties, which contain built-in molecules of various compounds ('guest' molecules, Fig. (2)), is the most important problem in nanodesign.

Two aspects related to problem of the "guest" molecules attract the attention. It is well-known that initial ds DNA molecules can easily "absorb" various chemical or biological molecules in the "free" space available inside the helical structure of the DNA molecules. For instance, this space is existing in the helical grooves on the DNA surface, and a socalled "groove-binders" are capable of fixing here. Besides, the partial unwinding of the DNA secondary structure "opens" a space between the DNA base pairs, and a so-called "intercalators" can occupy this space. Taking into account that the spatial ordering of the DNA molecules does not alter the parameters of their secondary structure, there is no principal difference in the concentrations of "guest" molecules absorbed by initial ds DNA in a "free" solution or by ds DNA in the content of the 3-D nanostructure. From this point of view, the creation of $\mathrm{NaC}$ 's according to "step-bystep" strategy capable of containing in their content a superhigh concentration of "guest" molecules seems to be illusive. However, there is another aspect of this problem, a namely, a free space available between neighboring NA molecules in the 3-D structure. This means, that theoretically there is a possibility to use this space for immobilization (accumulation) of diverse chemical compounds.

To form spatial NaC's with built-in guest molecules, Mirkin [19] and Letsinger et al. [20], as well as Alivisatos et al. [21] almost simultaneously used a procedure based on application building blocks in the form of synthetic singlestranded NA fragments linked to colloidal gold nanoparticles. If an alien NA, in which the sequence of nitrogen bases is complementary to the starting NA, is added to the abovementioned system, the hybridization leads to the formation of a rigid ds NA molecule. In this case, gold nanoparticles are located at a strictly fixed distance, which can be controlled accurate to $3.4 \AA$. Attaching a larger number of single-stranded NA fragments to the gold nanoparticles, one can form 3-D nanostructures, in which gold atoms alternate at regular intervals with ds NA molecules. 


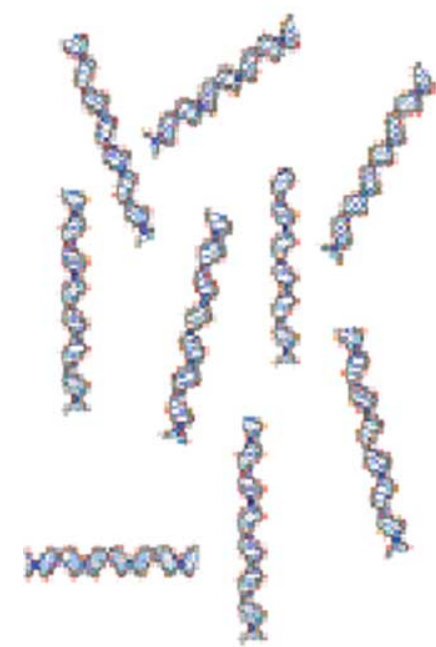

DNA molecules

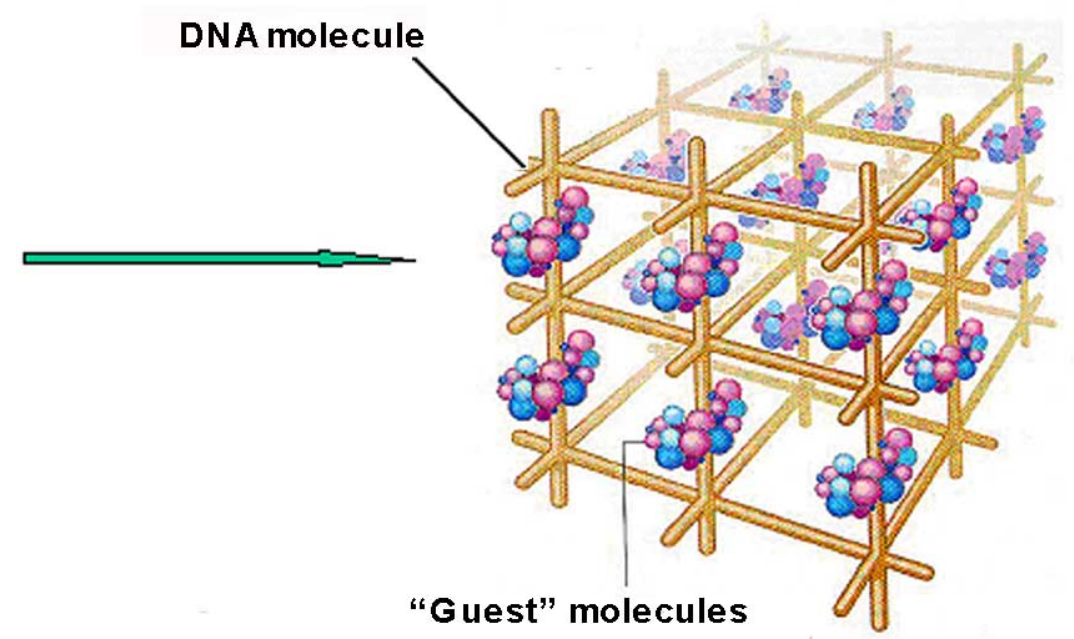

Fig. (2). Hypothetical nanostructure containing "guest" molecules (published with permission of the author, N. Seeman).

Different aspects of the formation and possible practical applications of NaC's composed of metal nanoparticles and oligonucleotide (nucleic acid) molecules were reviewed [22]. In addition, new approaches to design nanolattices containing various "linkers", such as carbon-nanotubes (CNT), etc. were described very recently [11-13].

Note that practical application of $\mathrm{NaC}$ 's formed from single NA molecules using the "step-by-step" technique is apparently determined by the kind of problems to be solved. The "guest" molecules in the composition of $\mathrm{NaC}^{2}$ can be targets for other biologically active compounds; i.e., biosensing units can be designed on the basis of $\mathrm{NaC}^{\prime}$ 's to detect materials recognizing "guest" molecules. In addition, the use of 2-D lattice composed of the DNA molecules (polynucleotides) as masks with subsequent incorporation of conducting materials (such as gallium arsenide or indium phosphide) into these networks opens, according to Mauro and Hollenberg [23], the possibility of design the elements (chips) for microelectronics with accuracy greatly exceeding the accuracy of conventional techniques. Insofar as there is no unequivocal answer to the question regarding the distance of electron transfer along the DNA chains, nanoelectronics cannot yet rely on the properties of a single DNA molecule alone. Hence, $\mathrm{NaC}$ 's combining classical nanoelectronic elements that are of a type with metal nanoparticles and the DNA molecules may show much promise.

Finally, if it were possible to perform 3-D ordering of individual spatial $\mathrm{NaC}$ 's (i.e. their crystallization), there might be a possibility of crystallizing in such a structure compounds that are poorly crystallized under conventional conditions but can be incorporated in a particular way into the $\mathrm{NaC}$ composition; i.e., it would be possible to perform a specific treatment of "guests". However, such ordering is a difficult problem that has not yet been solved.

In summary, we can say that, despite of definite achievements in the "step-by-step" strategy, i.e., in the use of individual ds NA molecules as building blocks for nanodesign, the question on formation of ordered 3-D spatial nanoconstructions with controlled properties based on complexes of these molecules, and practical application of such $\mathrm{NaC}$ 's remains open to a large extent.

The physical chemistry of ds NA molecules and their complexes allows to use a quite different approach for spatial ordering the neighboring NA molecules complexed with various compounds.

\subsection{Spontaneous Spatial Ordering Nucleic Acid Mole- cules}

There are two principally different ways (Fig. 3) for spontaneous ordering of ds NA molecules or their complexes with biologically relevant compounds. Specifically these ways determine the design of $\mathrm{NaC}^{\prime} \mathrm{s}$ of various types.

The first way (Fig. (3), I) is the "entropy" condensation; i.e., the process whose driving force is a change in the entropy of a system [24]. It is known that evaporation of aqueous salt solutions containing rigid, helical, ds NA molecules of low mol. mass (below $1 \times 10^{6} \mathrm{Da}$ ) results in spontaneous ordering these molecules, which leads to the formation of the liquid-crystalline (LC) phases of various types - from cholesteric to orthorhombic [25-27]. It should be noted that the tendency to cholesteric packing is a characteristic property of ds NA (DNA or RNA) molecules (determined by their geometric and optical anisotropy), which these molecules tend to implement first of all.

In addition to the liquid-crystalline phases, the liquidcrystalline dispersions (LCD's) of ds NA, which arise upon condensation of these molecules, are also known [28, 29]. Such condensation, realized at the phase exclusion of NA molecules from water-salt solutions by adding some polymers (in particular, polyethylene glycol (PEG)) to them, is referred to as the $\boldsymbol{\psi}$ - psi- (polymer-salt-induced) condensation (Fig. 4). As a result of phase exclusion, rigid ds NA (or polynucleotides) molecules form particles composed of about $10^{4}$ molecules; each particle is about $500 \mathrm{~nm}$ in size, which was theoretically evaluated using the results of several 


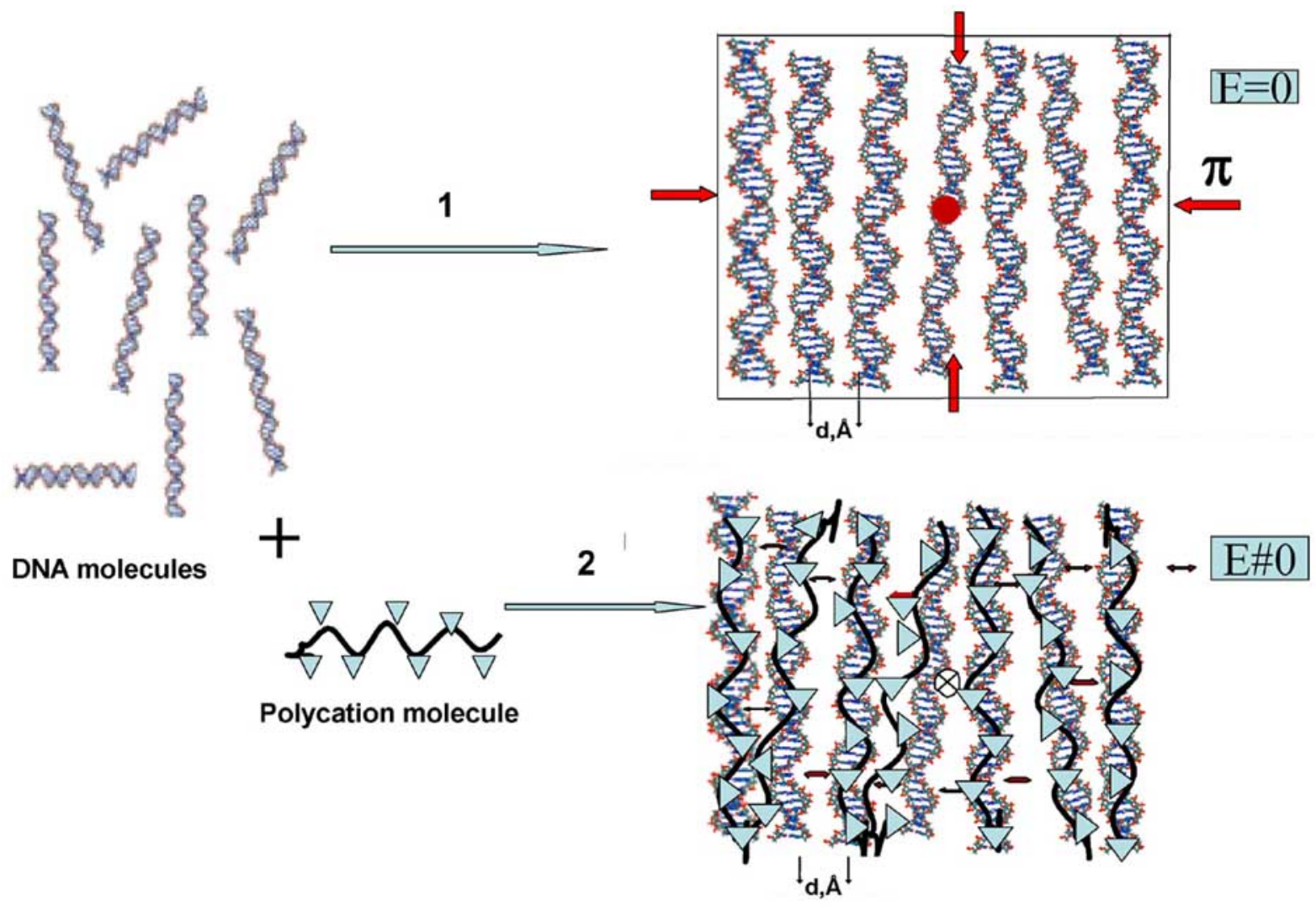

Fig. (3). Two ways for spontaneous ordering of double-stranded nucleic acid molecules. 1. the "entropy" condensation, as a result of phase exclusion of DNA molecules from water-salt polymer-containing solutions; $\mathbf{d}$ is a distance between neighboring "pure" DNA molecules; $\boldsymbol{\pi}$ means osmotic pressure of the solvent, and the energy (E) of interaction between DNA molecules is equal to zero in this case. 2 . the "enthalpy" condensation as a result of DNA interaction with biologically relevant polycation molecules; $\mathbf{d}$ is a distance between neighboring molecules of (DNA - polycation) complexes; and the energy (E) of interaction between neighboring molecules of (DNA - polycation) complexes, is not equal to zero in this case.

experimental techniques (low speed sedimentation, UV-light scattering, laser correlation spectroscopy, etc.) [28]. (Note, that in this case the polymeric molecules are not included in a composition of particles of LCD's). According to the X-ray study, NA molecules are ordered in the particle at distances of 3.0-5.0 nm, i.e. they acquire the properties of a crystal, but molecules in the neighboring layers are mobile, i.e. they retain the properties of a liquid. Such combination of properties allows this structure to be called as "liquid-crystalline" (see reviews $[25,29]$ and early references cited therein).

The most important features of ds NA LCD's are well established now. First, LCD's exist under certain boundary conditions, which are determined by solution ionic strength, by the value of osmotic pressure of water-salt-polymeric solution, etc. The osmotic pressure, which depends on polymer concentration in solution, determines the distance between neighboring NA molecules in a particle. Second, spontaneous constraint of diffusion degrees of freedom of neighboring NA molecules takes place upon phase exclusion. (One can stress here, that between neighboring NA molecules a "free" space is existing indeed, and this space is available for molecules biological or chemical compounds. The distance between NA molecules and "liquid"character of packing these molecules provide the conditions for a quick diffusion of many compounds (i.e. "guest" molecules) between the NA molecules in one layer as well as between the neighboring NA layers in the spatial structure of the LCD). Third, the combination of geometrical and optical anisotropy of NA molecules causes each next layer formed by NA molecules in the structure of the liquid-crystalline particle (a so-called "quasinematic layer") to be turned through a certain angle with respect to previous one, i.e. spatially twisted or a so-called "cholesteric" structure of the particle arises. (Violation of the boundary conditions results in the disappearance of this spatial structure of particle). Fourth, because NA molecules contain chromophores (nitrogen bases absorbing in the UV-region of the spectrum), the resulting cholesteric may be named as "colored" one. Since the bases are virtually perpendicular to the long axis of NA molecules forming adjacent layers in the structure of the cholesteric, theory [30] predicts an appearance of an intense (abnormal) band in the circular dichroism (CD) spectrum in the absorption region of nitrogen bases, which is indeed observed experimentally. It should be noted that theory [30] imposes no limitations on the number of colored compounds that could be incorporated into the LCD structure in the same manner, i.e. one could expect an appearance of the abnormal CD bands preferably for compounds located by the mode similar 


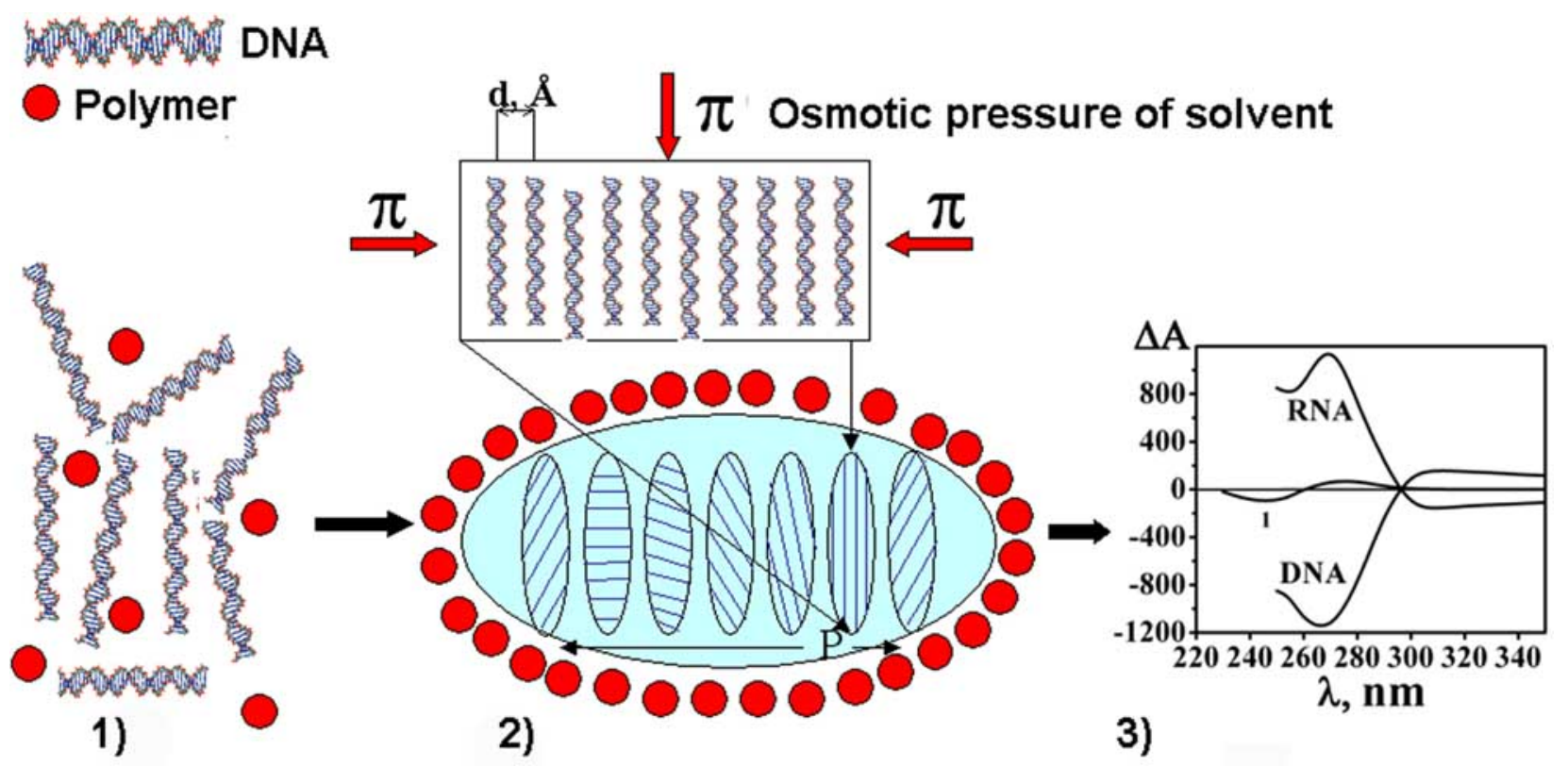

Fig. (4). Schematic diagram of the formation of a particle of a double-stranded nucleic acid cholesteric liquid-crystalline dispersion (CLCD). Polymer molecules are shown by filled circles (1) and the DNA CLCD particle is encircled by an oval (2), $\pi$ means osmotic pressure of solvent, ordering DNA molecules in one layer is shown. Note that polymer molecules do not enter the composition of nucleic acid LCD particles. The formation of CLCD particles characterized by spatially twisted packing of neighboring DNA (or RNA) molecules is accompanied by the appearance of an abnormal negative band in the CD spectrum in the case of DNA and abnormal positive band in the case of RNA in the absorption region of nitrogen bases (3).

to that one of nitrogen bases in respect to the ds DNA helix, i.e. especially for compounds incorporated (intercalated) between the NA nitrogen bases. This means that the CD band is an analytical "instrument" capable of monitoring the finest variations in the properties of NA molecules and cholesteric produced thereof. And finally, the chemical reactivity of NA molecules remains unchanged upon formation of the LCD particles; this opens the way for careful, directed alteration of the properties of these molecules.

The second mechanism of formation of LCD's is the "enthalpy" condensation (Fig. (3), 2). The driving force of this process is the attraction between neighboring NA molecules [31] whose negative charges of phosphate groups are neutralized by the counter ions of polycations. The most important peculiarities of the dispersions of (NA-polycation) complexes are shown in Fig. (3). First, the exclusion of the ds NA acid occurs when a critical concentration of polycation in the solution is reached (note that in this case, polycation molecules are always part of the composition of the particles formed). Second, the distance, $\mathbf{d}$, between the molecules of (NA- polycation) complex in the particles formed is fixed and generally close to $2.5 \mathrm{~nm}$. Third, in some "lucky" cases, depending on the properties (structure, charge distribution, etc.) of polycation molecules, an LCD can be formed from (NA-polycation) complexes, which is characterized not only by a fixed distance between molecules of (NA-polycation) complexes, but also by an abnormal CD band, i.e. it becomes possible to form a cholesteric LCD (see optical properties of the cholesteric LCD above). Finally, at formation of particles of an LCD of (NA-polycation) complexes, the initial reactivity of NA molecules can be significantly limited as a result of the steric location of polycation molecules on the surface of a NA molecule. However, reactivity caused by chemical groups of polycation molecules arises instead. This circumstance opens up the fundamental possibility of using such groups as new reactive centers with high diffusion availability.

Considering both ways for spontaneous ordering of ds NA molecules or their complexes one can conclude that, theoretically, a fundamental possibility to use NA molecules spatially fixed within the particles of LCD as precursors with adjustable properties for $\mathrm{NaC}$ formation is existing, i.e. it is possible to create $\mathrm{NaC}$, whose properties can be specified in advance by controlling both the properties of NA molecules and the solvent used. With CLCD particles (that is, a structure in which adjacent NA molecules are ordered and slow moving) in hand, there appears a possibility not only to use a free space between adjacent NA molecules for fixation here "guest" molecules, but to control optically the mode of the NA molecules packing.

Besides, despite the significant differences in both ways of NA molecules condensation (Fig. 3), ordered arrangement of (NA-polycation) in LCD particles and reactivity of chemical groups of polycation molecules can provide the possibility of fixation "guest" molecules as well.

Hence, for both cases, the fundamental problem is reduced to fixation of "guest"molecules between neighboring NA (or polycation) molecules located at a distance of 2.5 $5.0 \mathrm{~nm}$ in structure of CLCD particles. A possible approach for such a fixation consists in the formation of artificial cross-links between NA molecules. In so doing, it is necessary to retain the spatial arrangement of the particle (i.e. its abnormal optical activity). Taking into account the distances 
between NA molecules one can call the cross-links as "nanobridges" between NA (or polycation) molecules.

\subsection{All-At-Once Design}

The version of nanodesign strategy proposed by us in [2] is based on the use, instead of individual NA molecules, these molecules fixed in spatial structure of their particles of CLCD arising upon phase exclusion of NA molecules from water-salt solutions.

Obviously, formation of cross-links (nanobridges) between neighboring NA molecules requires the presence of terminal sites on the surface of NA molecules. If such sites are located on neighboring NA molecules, a nanobridge will cross-link the neighboring NA's. Theoretically, such a terminal site can be metal ions specifically fixed in a groove (grooves) on the NA surface capable both of forming chelates with NA nitrogen bases and attaching other ligands that are additionally introduced into the system. Besides, molecules of planar compounds also tend to form the external reaction-able complexes with NA molecules. In view of these circumstances, antibiotics of the antracycline group are of interest. These compounds, owing to their chemical structure, can form chelate complexes with ions of divalent metals, in particular, with $\mathrm{Cu}^{2+}$ ions. Chelate complexes may contain up to 10 repeating subunits; i.e., under certain conditions, antracyclines can form extended planar structures with $\mathrm{Cu}^{2+}$ ions (polymeric chelate complexes [32]). Antibiotics of this group (in particular, daunomycin (DAU)) can intercalate between pairs of bases of nucleic acids and synthetic polynucleotides only of the B-family. Upon intercalation, the reactive DAU groups (keto-oxygen and peri-OH-groups) are inaccessible for the chemical reactions. However, it is known that DAU and its analogs can form so-called "external" complexes with the nucleic acids both of the B- and Afamilies [33]. As a result of the formation of an external complex, reactive DAU oxygen-groups become available for chemical reactions, in particular, for chelate formation. Therefore, DAU molecules can also play the role of terminal sites for nanobridges.

Thus, this nanodesign technology is based on the combination of several known experimental results and some theoretical possibilities, specifically: spatial ordering of neighboring, existence of free space between closely packed NA molecules in cholesteric LCD particles, the relative limitation of NA molecules diffusion mobility, the formation of polymer chelate complexes from alternating metal ions and antracycline molecules under conditions of the phase exclusion of NA molecules, and the use of copper ions and (or) antracycline molecules as terminal sites for nanobridges.

A nanoconstruction was obtained according to the following scheme: a CLCD was formed from ds NA molecules (Fig. (5), curve 1), a DAU solution was added to it up to the formation of a external complex (curve 2), and then the dispersion was treated with a $\mathrm{CuCl}_{2}$ solution (curve 3 ). Comparing the curves in Fig. (5), one should bear in mind that the abnormal band in the absorption region of DNA $(\lambda \sim 270$ $\mathrm{nm}$, curve 1) reflects the cholesteric quality: the more native the secondary DNA structure and the more ordered the cholesteric packing of DNA molecules, the stronger this band. The amplitude of this band depends linearly on the DNA concentration: the higher the DNA concentration used in the preparation of the LCD, the stronger the band. Addition of $\mathrm{CuCl}_{2}$ to the DNA CLCD processed with DAU and having an equilibrium value of the band amplitude at $\lambda \sim 500 \mathrm{~nm}$ (curve 2) leads to the amplification of this band related to the optical properties of the chromophore of the $\left[\mathrm{DAU}-\mathrm{Cu}^{2+}\right.$ ] complex. All other factors being equal, the amplitude of this band increases with the increase in the amplitude of the DNA abnormal negative CD band at $270 \mathrm{~nm}$. Under the conditions used (the DNA molar mass is $8 \times 10^{5} \mathrm{Da}$ and the DNA concentration is $5 \mu \mathrm{g} / \mathrm{ml}$ ), the maximum amplitude of the band at $\lambda 500 \mathrm{~nm}$ is equal to 2500 (in $\Delta \mathrm{A}$ units).

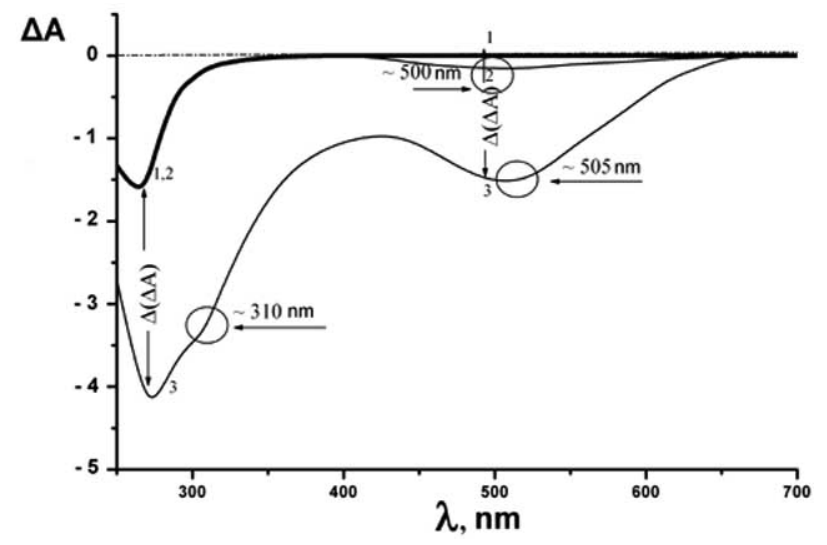

Fig. (5). CD spectra of (1) the initial DNA CLCD and the CLCD DNA successively processed by (2) $\mathrm{DAU}$ and (3) $\mathrm{CuCl}_{2}$ solutions. $\Delta \mathrm{A}=\mathrm{A}_{\mathrm{L}}-\mathrm{A}_{\mathrm{R}}$, opt. units $\left(\times 10^{-3}\right)(\Delta(\Delta \mathrm{A})$ is increase in the band amplitude with the subtracted value of $\Delta \mathrm{A}$ that is characteristic of the initial DNA LCD).

According to the theory [30], the amplification of the band in the chromophore absorption region shows that the chromophores of the [DAU- $\mathrm{Cu}^{2+}$ ] complexes are spatially fixed with respect to the NA molecules in the composition of CLCD particles. Although there are two different models of fixation of $\left[\mathrm{DAU}-\mathrm{Cu}^{2+}\right]$ chelate complexes near the surface of NA molecules, the experimental data are unambiguously in favor of the model of the complex playing the role of a nanobridge between nucleic acid molecules [2].

The prerequisites for formation of nanobridges (i.e. the structure of anthracycline compounds, the "phasing" and distance between ds NA molecules, etc.) clearly show that the creation of these nanobridges is a very delicate stereochemical process, which can be realized only under rather strict conditions [2]. The number of $\mathrm{Cu}^{2+}$ ions in the content of nanobridges was estimated directly [34]. If $\mathrm{Cu}^{2+}$ ion forms a chelate complex with four reactive oxygen atoms; this ion is in a $\mathrm{d}^{9}$ state, which exhibits nonzero magnetic moment [34]). The low-temperature magnetometry, that was used for evaluation of the total magnetic moment, demonstrates that a nanobridge contains six copper ions (II) and a $\left[--\mathrm{Cu}^{2+}-\right.$ DAU - $\mathrm{Cu}^{2+}-\ldots-\mathrm{Cu}^{2+}$-DAU $\left.-\mathrm{Cu}^{2+}-\right]$ nanobridge between neighboring NA molecules has the structure shown in Fig. (8).

The efficiency of the formation of nanobridges depends on the concentrations of both DAU molecules and copper ions. Despite of different values of the band amplitudes in the UV- and visible regions of the CD spectrum, the obtained dependencies are S-shaped with a high degree of consistency. (Amplification of the bands begins when the criti- 
cal concentrations of both copper ions and DAU molecules are attained in the solution). For DAU molecules, the critical concentration means that the DAU molecules form external complexes, which arise after the DAU intercalation, are involved in the formation of nanobridges. The critical concentration in the case of copper ions means that these ions induce some changes in the NA molecule, after which copper ions (or their complexes with pairs of bases and DAU molecules) become available for further chelating that is necessary for nanobridge formation.

The amplitudes of the abnormal bands in the CD spectrum both in the visible and UV- regions of the CD spectrum decrease with an increase in temperature. It is characteristic that the amplitude of the band in the visible region (at $\lambda 505$ $\mathrm{nm}$ ) decreases almost to zero, whereas the UV band (at $\lambda 270$ $\mathrm{nm}$ ) decreases to a value corresponding to the amplitude of the band of the initial DNA CLCD (Fig. (5), curve 1). This fact means that, at a fixed osmotic pressure of the solution (set by the PEG concentration), the CLCD structure remains unchanged under the heating conditions used. The temperature dependences of the amplitudes of the CD bands of a DNA CLCD containing nanobridges at different DAU and $\mathrm{CuCl}_{2}$ concentrations are shown in Fig. (6). Comparison of these curves indicates that an increase in the concentration of the nanobridge components and, therefore, the number of nanobridges in the composition of an LCD particle, leads to a few effects: the amplitude of the abnormal CD band is increased and the shape of the curve is changed. Indeed, at a low concentration of the nanobridge components (curve 1), the temperature dependence is exponential, whereas, at their high concentration (curve 3), the dependence becomes Sshaped. Finally, with a growth of the concentration of nanobridges, the temperature at which nanobridges are destroyed, is increased. This circumstance speaks in favor of stabilization of the structure of DNA CLCD particles.

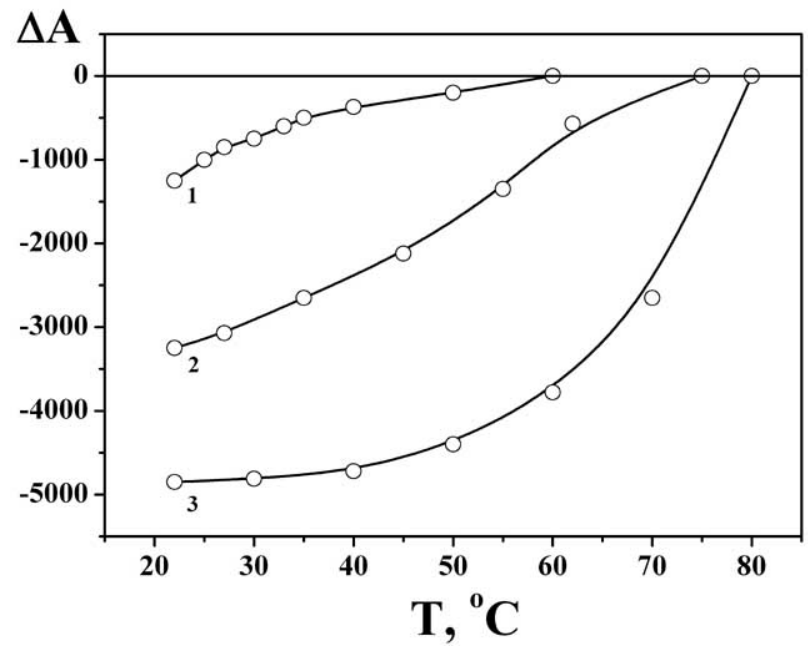

Fig. (6). Temperature dependences of the amplitudes of the bands $(\lambda 310 \mathrm{~nm})$ in the CD spectrum of DNA LCD's containing nanobridges at different $\mathrm{DAU}$ and $\mathbf{C u C l}_{\mathbf{2}}$ concentrations. $\mathrm{C}_{\mathrm{DAU}}$ $\times 10^{-6}=$ (I) 15 , (2) 27.3 , and (3) $64 \mathrm{M} ; \mathrm{C}_{\mathrm{Cu}}{ }^{2+} \times 10^{-6}=$ (1) 5, (2) 9.9 , and (3) $20 \mathrm{M} ; \mathrm{C}_{\mathrm{PEG}}=170 \mathrm{mg} / \mathrm{ml} ; 0.3 \mathrm{M} \mathrm{NaCl} ; 0.002 \mathrm{M} \mathrm{Na}^{+}$phosphate buffer; $\mathrm{pH}=6.7 ; \Delta \mathrm{A}=\mathrm{A}_{\mathrm{L}}-\mathrm{A}_{\mathrm{R}}$, opt. units $\left(\times 10^{-3}\right)$.

The increase in the melting temperature of nanobridges with an increase in the concentration of the structural ele- ments of nanobridges shows that a new nanostructure arises in the content of cholesteric structure of DNA LCD particles. This nanostructure is composed of nanobridges located both within and between layers of neighboring DNA molecules.

To determine the state of nucleic acid molecules in CLCD particles, X-ray diffraction analysis of the phases obtained from CLCD's was performed [2]. The X-ray scattering curves contain only one maximum in the range of angles $1^{0}<2 \theta<5^{0}$, which corresponds to the average distance between molecules in LCD particles. The value of $\mathbf{d}_{\text {Bragg }}$ for DNA molecules fixed in the spatial structure of LCD particles formed of the [DNA-DAU] complex processed in a $\mathrm{CuCl}_{2}$ solution (i.e., between the DNA molecules crosslinked by nanobridges) is $30.1 \AA$. The absence of high-order reflections in the X-ray diffraction patterns indicates the absence of regular 3-D order in the arrangement of molecules of the [DNA-DAU] and [DNA-DAU- $\mathrm{Cu}^{2+}$ ] complexes in LCD particles; therefore, we can only speak about the existence of short-range order in the arrangement of molecules in these particles.

The high local DNA concentration in CLCD particles and DNA LCD particles containing nanobridges corresponds to the concentration range in the phase diagram of DNA, where these molecules are in the cholesteric liquidcrystalline state [26,29].

Comparison of the results of $\mathrm{X}$-ray diffraction analysis with the above data on melting of nanobridges indicates that the formation of nanobridges between NA molecules does not violate the initial spatial packing of NA molecules; moreover, it leads to its stabilization. This allows one to suggest that there are conditions, under which this structure must maintain itself even in water-salt solution. In this case appears a possibility to investigate the properties of this structure not only by theoretical, but experimental techniques, for instance, it can be visualized (Fig. 7). Indeed, immobilization of the DNA LCD particles treated by DAU and then by $\mathrm{CuCl}_{2}$ on a nuclear membrane filter and investigation of these particles by AFM shows that every particle is a 3-D construction. The particles are shaped as elongated cylinders. Estimation of the size of 400 particles demonstrates that the sizes varied from $0.3 \mu \mathrm{m}$ to $0.8 \mu \mathrm{m}$ with 0.5 $\mu \mathrm{m}$ as the average, which is in good agreement with the data on the particle size calculations for the initial DNA CLCD's obtained by other techniques [2]. This means that, as a result of the formation of nanobridges between neighboring DNA molecules fixed in the spatial structure of CLCD particles, a spatial structure of CLCD particles arises, in which the neighboring DNA molecules are "frozen". With allowance for the fact that nanobridges can arise both between neighboring DNA molecules of the same quasinematic layer and between DNA molecules of neighboring layers, the formation of nanobridges, in principle, means that, instead of the osmotic pressure of a PEG-containing solution, another factor of the spatial structure stabilization arises, specifically, the number and stability of nanobridges.

Thus, not only the particles nanodesigned on the basis of DNA CLCD's have been visualized but also some data characterizing the macroscopic parameters of these particles have been obtained. 
(b)

(a)

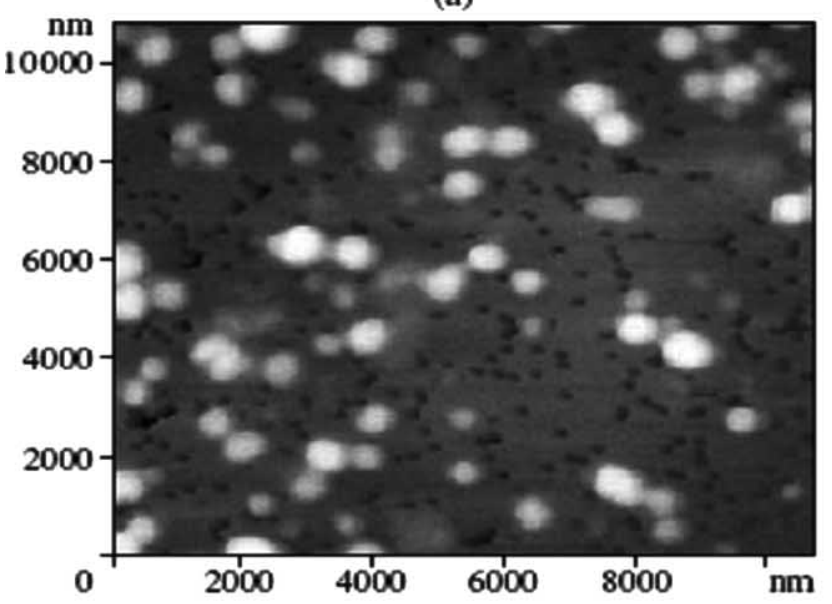

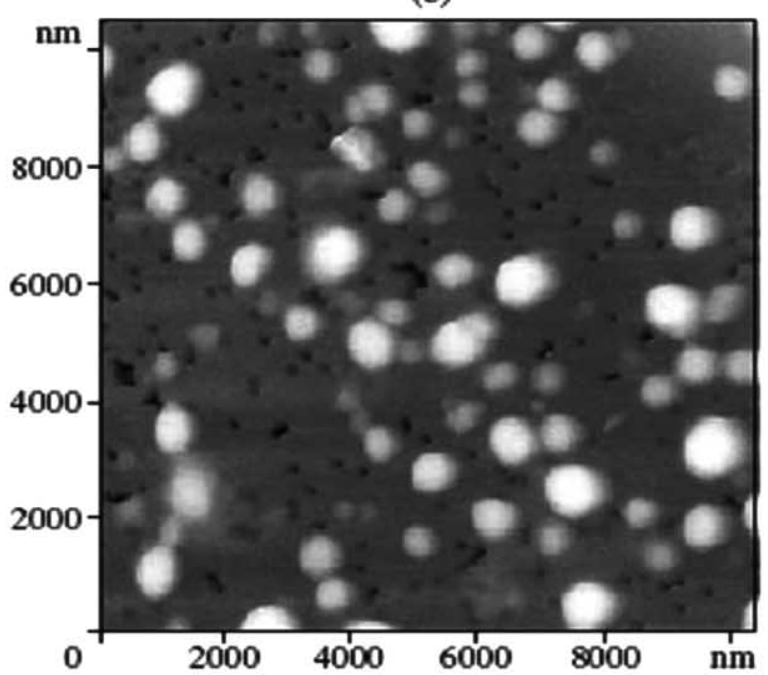
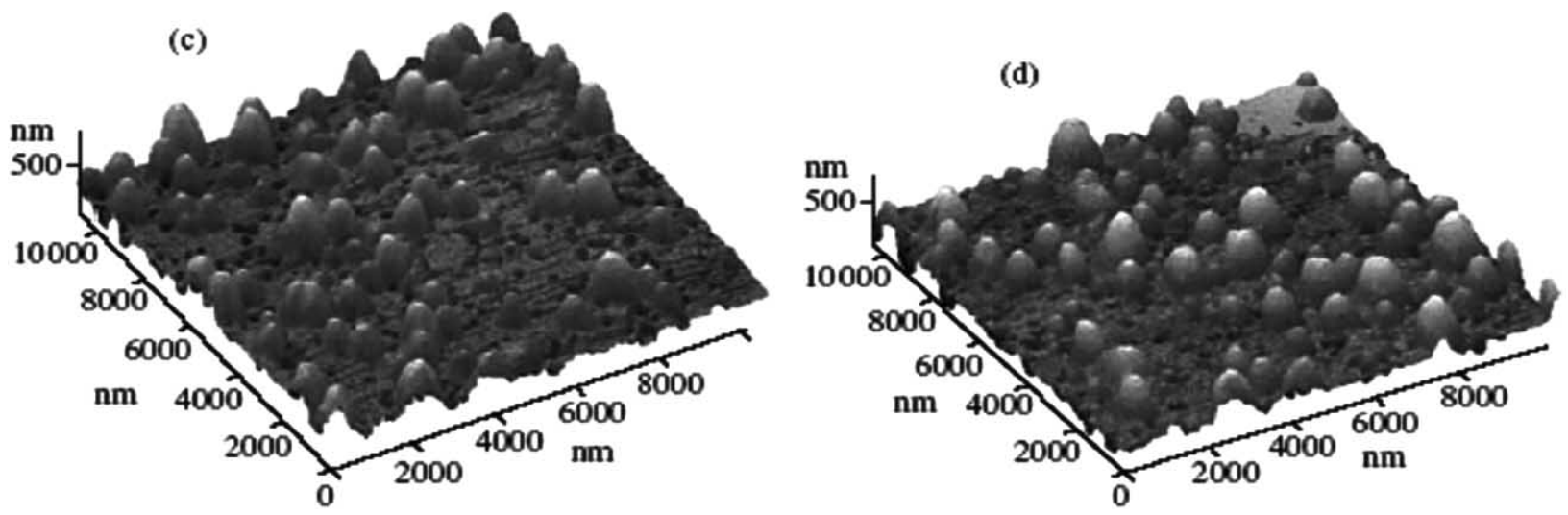

Fig. (7). 2-D and 3-D images of DNA particles cross-linked by nanobridges and immobilized on a nuclear membrane filter (a), (b) and (c), (d), respectively. (Dark spots are holes in the filter).

There are a few principal differences between initial particles of the NA LCD's and NA NaC's. First, in contrast to the NA LCD, the structure of $\mathrm{NaC}$ is not "liquid-crystalline" any more; rather it is a rigid, crystal-like structure. Indeed, $\mathrm{X}$-ray diffraction analysis indicates a higher degree of crystallinity of the DNA NaC's as compared to the particles of DNA LCD. Second, in contrast to the NA LCD, NaC has extraordinary high optical activity both in the UV- and visible regions of the $\mathrm{CD}$ spectrum. Third, $\mathrm{NaC}$ may consist (probably) of two cholesterics. Along with a cholesteric composed of initial NA molecules, there is a possibility for formation of a cholesteric from the nanobridges located between neighboring NA layers. This means that, in the process of formation of the $\mathrm{NaC}$, the abnormal optical activity in the absorption band of the antibiotic $(\lambda \sim 500 \mathrm{~nm})$ rises dramatically, enabling us to observe minor changes in the properties of the $\mathrm{NaC}$ designed. Theoretical analysis of the properties of such structures was started recently [35].

The fact of existence of a fairly stable structure whose properties depend on the properties of nanobridges allows one to propose the structure of a $\mathrm{NaC}$ based on DNA CLCD particles (Fig. 8). In this $\mathrm{NaC}$, neighboring DNA molecules form layers with nanobridges composed of alternating antibiotic molecules and metal ions and located within and between these layers. This $\mathrm{NaC}$ is a 3-D formation; the diffusion mobility of neighboring NA molecules in it is fairly low and, therefore, this structure does not possess many properties characteristic of NA CLCD's. The NaC developed is unique because it consists not only NA molecules but also antibiotic molecules and copper ions, i.e. "guest" molecules. Besides, this structure accommodates a high concentration not only of NA molecules alone (up to $400 \mathrm{mg} / \mathrm{ml}$ ) but antibiotic molecules (about $200 \mathrm{mg} / \mathrm{ml}$ ) and copper ions. This fact indicates that the use of CLCD particles for nanodesign automatically solves the problem that remains unsolved in the "step-by-step" strategy, specifically, ordering of both neighboring NA and "guest" molecules. In addition, the cholesteric structure is formed not only by NA molecules but also "guests" (antibiotic molecules and copper ions). This fact shows that the formation of $\mathrm{NaC}$ sharply increases the abnormal optical activity in the absorption region of the antibiotic, a circumstance that is very important from the practical point of view since such a high optical activity makes it possible to monitor with high accuracy all changes in the properties of the $\mathrm{NaC}$ formed. Concerning the scientific 


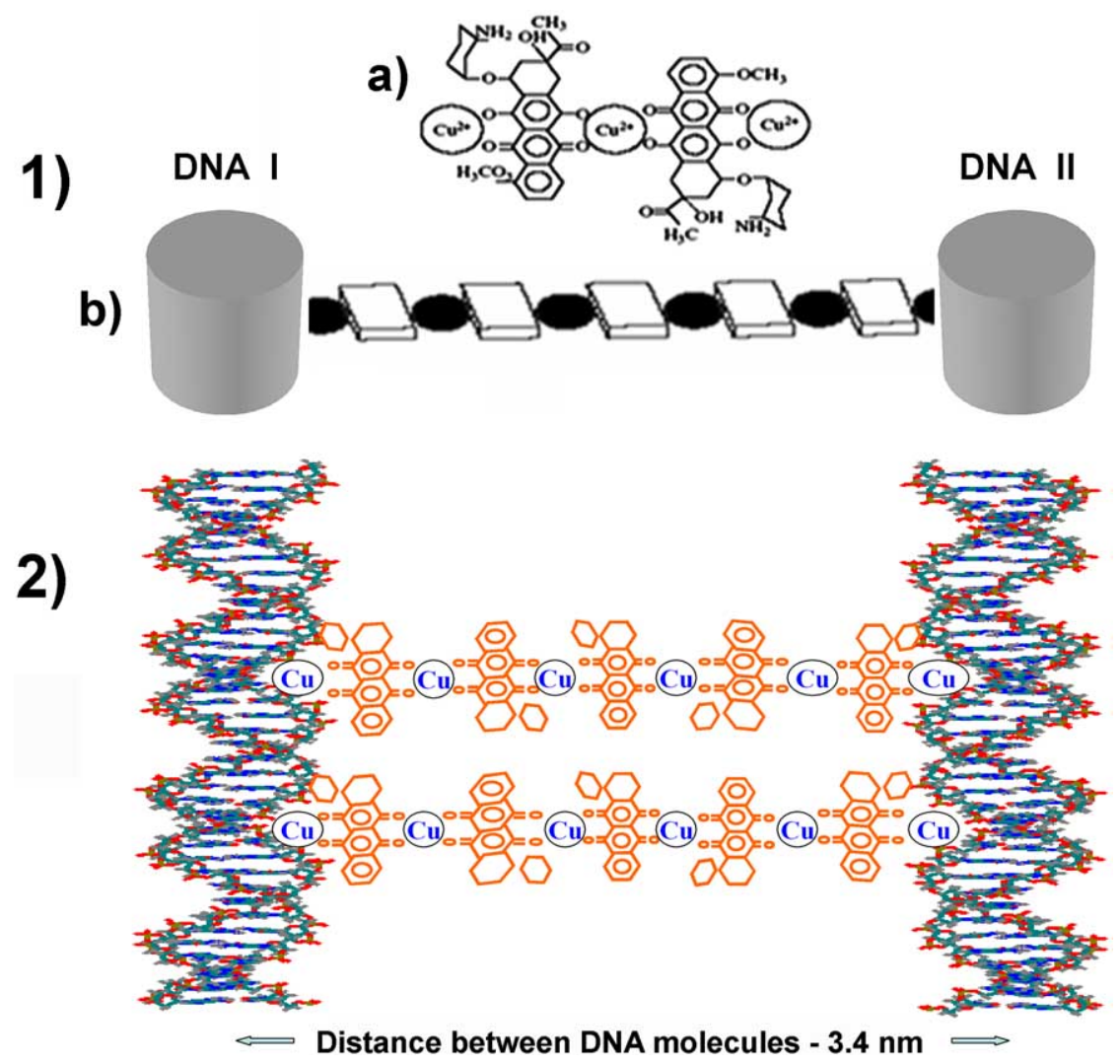

Fig. (8). Structure of nanobridges formed between DNA molecules fixed in spatial structure of CLCD particles. 1). a). Structure of chelate complex between daunomycin molecules and cupper ions. b). Schematic top view of nanobridge between DNA (I) and DNA (II) molecules. 2). Nanobridges between DNA molecules (for simplicity, nanobridges are turned on $90^{\circ}$ degrees in respect to their standard position).

value of this $\mathrm{NaC}$, we can say that a new structure based on a NA CLCD has been obtained, which was unknown previously. $\mathrm{NaC}$ is a nanomaterial of a new type, whose properties can be controlled in accordance with customer's demands.

Despite of the "solid" (rigid) structure of $\mathrm{NaC}$, many compounds (from ascorbic acid to poly(aminoacids) and peptides with molecular masses up to $60 \mathrm{kDa}$ ) diffuse inside the spatial structure of $\mathrm{NaC}$ very easily.

The considered nanodesign approach may be further improved. In particular, other chemicals or biologically active compounds, as well as their complexes, may be used for nanobridges, or the surface of NA molecules may be chemically modified to diversify the reactivity and afford new types of $\mathrm{NaC}$ 's.

An attempt to form a $\mathrm{NaC}$ using the procedure based on the "enthalpy condensation" (as a result of DNA molecules interaction with polycation) was described in [36].

It was shown above, that only in some "lucky" cases, depending on the properties of polycation molecules, CLCD can be formed from (DNA-polycation) complexes, which is characterized by certain distance between molecules of (DNApolycation) complexes, and an abnormal CD band. Hence, for this case the choice of polycation molecule is a crucial point. From this point of view, natural, biodegradable, biocompatible polyaminosaccharide - chitosan (Chi, poly- $\beta$-(1-4)-2-amino-2deoxy-D-glucopyranose) seems to be promising [37]. In view of the specific features of the spatial structure of Chi molecules, positively charged Chi amino groups can alternately interact with negatively charged DNA phosphate groups. Chi molecules interact with DNA in such a way as to allow amino groups of Chi sugar residues not only neutralize the negative charges of DNA phosphate groups but also form a specific distribution of positively charged free amino groups near the DNA surface. Since neighboring free amino and hydroxyl groups in Chi molecules form effectively chelate complexes with copper ions [37-40], to perform cross-linking of neighboring Chi molecules fixed in the structure of DNA-Chi CLCD particles, the same approach that had been used to form nanobridges between pure DNA molecules was applied.

A CLCD was formed from ds DNA molecules via their interaction with $\mathrm{Chi}$; then DAU (or $\mathrm{CuCl}_{2}$ ) solution was added to it; and, finally, processing in a $\mathrm{CuCl}_{2}$ (or DAU) solution was performed. Fig. (9) shows that addition of $\mathrm{CuCl}_{2}$ to a (DNA-Chi) CLCD (this dispersion exhibits an abnormal positive band in the CD spectrum) and its subsequent treatment in DAU leads to manifold amplification of a positive band at $\lambda \sim 500 \mathrm{~nm}$, which corresponds to the optical properties of the chromophore of the [DAU- $\mathrm{Cu}^{2+}$ ] complex. Similar amplification does not depend on the order of addition of $\mathrm{CuCl}_{2}$ and DAU to the (DNA-Chi) CLCD. In particu- 
lar, such a treatment of the CLCD formed of DNA-Chi complexes (in which Chi molecules have another content of amino groups) and characterized by an intense negative band in the CD spectrum, the negative band in the region of DAU absorption in the CD spectrum is amplified as well.

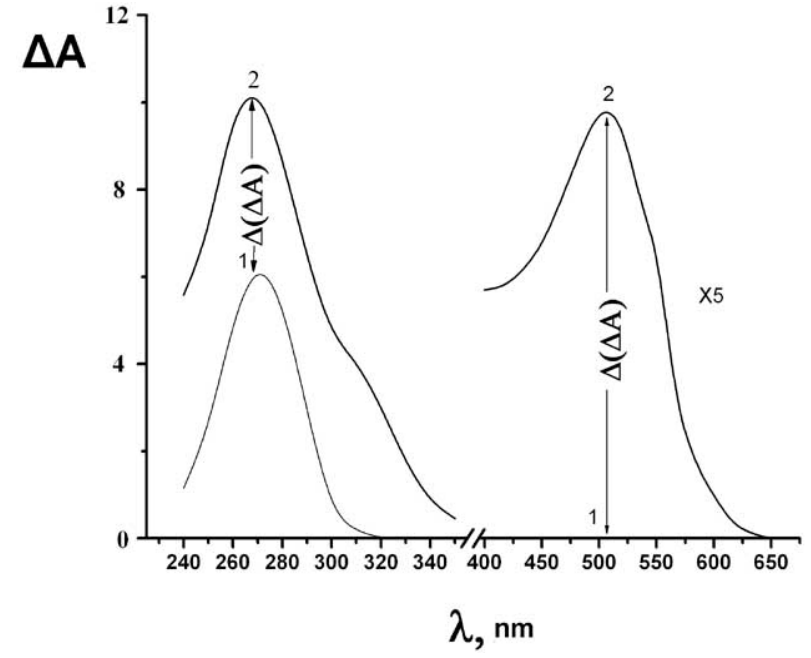

Fig. (9). (1) - UV and visible spectra of a CLCD of the DNAChitosan complex that was treated with $\mathrm{CuCl}_{\mathbf{2}}$ and then with daunomycin (2). $\mathrm{C}_{\mathrm{DNA}} \sim 15.5 \mu \mathrm{g} / \mathrm{ml}$; the Chi preparation contains $65 \% \mathrm{NH}_{2}$ amino groups, $5 \mathrm{kDa} ; \mathrm{C}_{\mathrm{DAU}} \times 10^{-6}=(1) 0$ and (2) $59.7 \mathrm{M}$; $\mathrm{C}_{\mathrm{Cu}}{ }^{2+} \times 10^{-6}=(1) 0$ and (2) $15 \mathrm{M} ; 0.05 \mathrm{M} \mathrm{NaCl} ; 0.002 \mathrm{M} \mathrm{Na}^{+}$phosphate buffer, $\mathrm{pH} 6.7 ; \Delta \mathrm{A}=\mathrm{A}_{\mathrm{L}}-\mathrm{A}_{\mathrm{R}}$, opt. units $\left(\times 10^{-3}\right)$.

Amplification of the band in the absorption region of the $\left[\mathrm{DAU}-\mathrm{Cu}^{2+}\right]$ chromophore reflects, according to the theory [30], that the spatial fixation of this chromophore with respect to Chi molecules in any CLCD formed by various DNA-Chi complexes.

The important difference of a (DNA-Chi) CLCD from a pure DNA CLCD is that the order of addition of DAU and $\mathrm{CuCl}_{2}$ solutions does not affect the extent of the amplification of the CD band. The efficiency of amplification of the abnormal band in the CD spectrum of (DNA-Chi) CLCD's depends also, as in the case of pure DNA, on the concentration of DAU molecules and copper ions. Some part of DAU molecules can intercalate between pairs of DNA bases in the composition of the (DNA-Chi) complex, but this process rapidly reaches an equilibrium. However, only after the end of intercalation an external DAU complex is formed (which is available for chemical reactions) and the abnormal band begins to sharply increase when a copper salt is added. The absence of the critical concentration in the case of copper ions indicates that, in contrast to the case of pure DNA, free chemical groups that are sterically available for chelate formation with copper ions are always present in Chi molecules in the structure of the (DNA-Chi) complex. Such free groups can be amino groups and hydroxyl groups of Chi sugar residues, which form chelate complexes with copper ions characterized by a large binding constant (about (1-4) x $10^{14} \mathrm{M}$ ) $[38,39]$.

A comparison of the data obtained (Fig. 9) with the data on the formation of nanobridges in the case of pure DNA (Fig. 5) demonstrates their qualitative coincidence. Hence, we can conclude that the amplification of the band at $\lambda \sim 500$ $\mathrm{nm}$ in the CD spectrum of CLCD's of different (DNA-Chi) complexes is related to the formation of nanobridges of the [$\mathrm{Cu} 2+-\mathrm{DAU}-\mathrm{Cu} 2+-]$ type between neighboring Chi molecules that are bound into complexes with DNA molecules in CLCD particles. With allowance for the fact that, as in the case of pure DNA, nanobridges are be formed both between neighboring Chi molecules within the same layer and between neighboring Chi molecules belonging to neighboring layers, the amplification of the band at $\lambda \sim 500 \mathrm{~nm}$ reflects the formation of a spatial $\mathrm{NaC}$. This fact shows that the formation of [$\mathrm{Cu} 2+-\mathrm{DAU}-\mathrm{Cu} 2+-]$ nanobridges (and, therefore, $\mathrm{NaC}$ 's based on the (DNA-Chi) CLCD) has the same optical manifestation in all cases. From this point of view, it is of interest to compare the maximal band amplitudes observed at the formation of a NaC based on CLCD's of pure DNA and (DNA-Chi) complexes. Under the conditions used (the DNA mol. mass is $8 \times 105 \mathrm{Da}$ and DNA concentration is $15 \mu \mathrm{g} / \mathrm{ml}$ ), the maximum amplitude of the band at $\lambda \sim 500 \mathrm{~nm}$ that is characteristic of (DNA-Chi) CLCD's does not exceed $2500 \Delta \mathrm{A}$ units, despite of the use of different Chi preparations. Comparing this value with the amplitude given in Fig. (5) and taking into account the correlation between the DNA concentration and the abnormal band amplitude in the CD spectrum of the CLCD, one can conclude with reasonable confidence, that in the case of (DNA-Chi) CLCD, the maximal band amplitude is smaller than that characteristic of NaC's formed of pure cholesteric ds DNA LCD's by a factor of about 3 . Such a difference may be caused by several reasons: (i) a smaller number of nanobridges; (ii) a smaller physical size of nanobridges (the distance between molecules in DNA-Chi complexes is smaller than that of the distance between pure DNA molecules; and (iii) the difference between the angle of inclination of nanobridges between Chi molecules in (DNA-Chi) complexes in forming CLCD's and the angle of inclination of nanobridges between pure DNA molecules. The latter reason may be related to the conformation of Chi molecules located at neighboring DNA molecules. Further investigations should clarify the specific reason for the above-mentioned differences.

Nevertheless, the fact that the physical size of nanobridges can be small in the case of (DNA-Chi) CLCD's, in combination with the ability of copper ions to form very strong chelate complexes with neighboring amino and hydroxyl groups of Chi sugar residues [38-41], suggests that the structure and properties of $\mathrm{NaC}^{\prime}$ 's obtained may significantly differ from the NaC's based on the pure DNA.

In particular, Fig. (10) shows that the amplitude of the abnormal band $(\lambda \sim 505 \mathrm{~nm})$ in the CD spectra of CLCD's of $\mathrm{NaC}$ 's based on different (DNA-Chi) complexes almost does not change with an increase in temperature. This fact indicates that the spatial structure of (DNA-Chi) CLCD remains invariable, i.e., the thermal stability of the NaC's based on (DNAChi) CLCD's significantly exceeds the stability of not only (DNA-Chi) CLCD's (curves 1,2) but also of the NaC's based on the pure DNA.

An interesting result, which directly speaks in favor of the importance of the conformation of Chi molecules for nanodesign, is the dependence of the amplitude of the abnormal band at $\lambda \sim 505 \mathrm{~nm}$ in the CD spectrum of (DNA- 


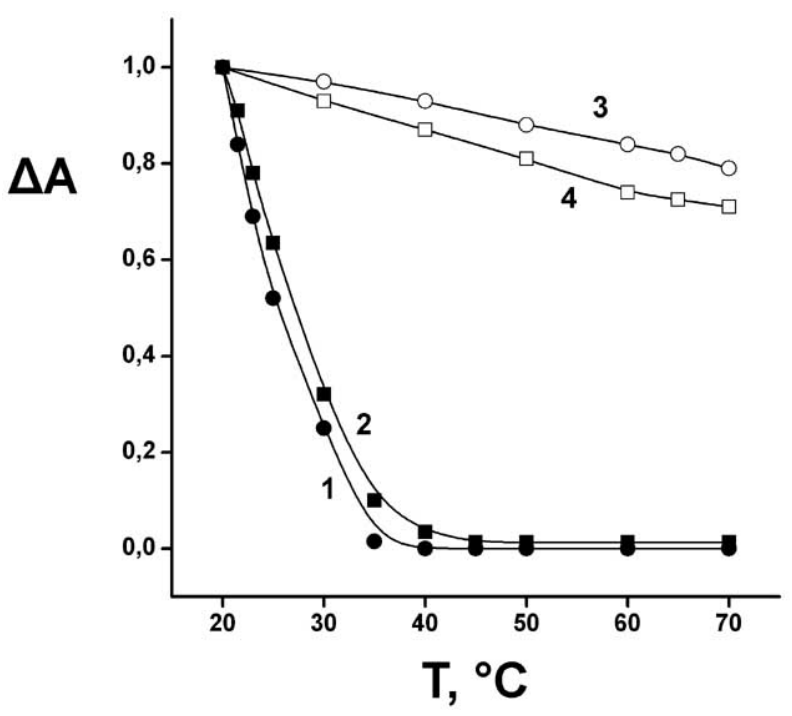

Fig. (10). Temperature dependences of the relative amplitudes of the bands in the CD spectra of CLCD's formed by (1) DNA-Chi 65 and (2) DNA-Chi 55 complexes $(\lambda=270 \mathrm{~nm})$ and $(3,4)$ nanoconstructions based on CLCD's of these complexes $(\lambda=505 \mathbf{~ n m})$. $\mathrm{C}_{\text {DNA }} \sim 15.5 \mu \mathrm{g} / \mathrm{ml}$; (1) Chi $65 \% \mathrm{NH}_{2} ; 5 \mathrm{kDa} ; 28.7 \mu \mathrm{g} / \mathrm{ml}$; (2) Chi $55 \% \mathrm{NH}_{2} ; 5 \mathrm{kDa} ; 28.7 \mu \mathrm{g} / \mathrm{ml}$; (3) $65 \% \mathrm{NH}_{2} ; 5 \mathrm{kDa} ; 28.7 \mu \mathrm{g} / \mathrm{ml}$; $\mathrm{C}_{\mathrm{Cu}}{ }^{2+} \times 10^{-6}=15 \mathrm{M} ; \mathrm{C}_{\mathrm{DAU}} \times 10^{-6}=50 \mathrm{M}$; and (4) Chi $55 \% \mathrm{NH}_{2} ; 5$ $\mathrm{kDa} ; 28.7 \mu \mathrm{g} / \mathrm{ml} ; \mathrm{C}_{\mathrm{Cu}}{ }^{2+} \times 10^{-6}=15 \mathrm{M} ; \mathrm{C}_{\mathrm{DAU}} \times 10^{-6}=50 \mathrm{M} ; 0.05 \mathrm{M}$ $\mathrm{NaCl} ; 0.002 \mathrm{M} \mathrm{Na}^{+}$phosphate buffer; $\mathrm{pH}$ 6.7.

Chi) CLCD's on the content of amino groups in the Chi molecules (Fig. 11). This dependence shows that there is a

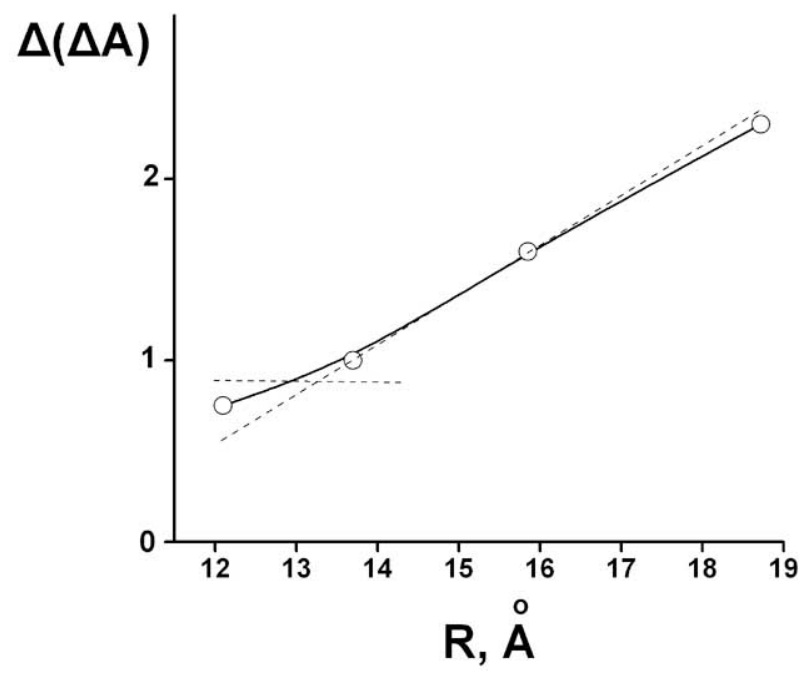

Fig. (11). Dependence of the maximal amplitude of the band at $\lambda=505 \mathrm{~nm}$ in the CD spectrum of nanoconstructions based on CLCD's of the DNA-Chi complexes on the average distance, $R$, between amino groups in Chitosan molecules. $\mathbf{R}(\stackrel{\AA}{\mathbf{A}})=2 \times(5.15$ $\times 100 \%) / \mathrm{NH}_{2}$; where $\mathrm{NH}_{2}$ is the percentage of amino groups in a Chitosan sample; $\mathrm{C}_{\mathrm{DNA}} \sim 15.5 \mu \mathrm{g} / \mathrm{ml} ; \mathrm{C}_{\mathrm{DAU}} \times 10^{-6}=55 \mathrm{M}$.

minimal distance between amino groups (about $13 \AA$, which approximately corresponds to the every-second-one distance) at which neighboring nanobridges composed of molecules of $\left(\mathrm{DAU}-\mathrm{Cu}^{2+}\right.$ ) complexes can be located. In this case, the low abnormal optical activity indicates that, despite of the high concentration of amino and hydroxyl groups in Chi sugar residues (potential sites of nanobridge formation), the spatial orientation of nanobridges formed is not optimal. Only with an increase in the distance between amino groups in Chi molecules, the steric structure of the Chi ensures the orientation of amino and hydroxyl groups in sugar residues that is appropriate for the formation of optimal nanobridges in the structure of the $\mathrm{NaC}$ formed.

Thus, comparison of the results obtained with the data on the nanodesign based on the pure DNA suggest that nanobridges can be formed between neighboring Chi molecules in (DNA-Chi) complexes that are fixed in the spatial structure of CLCD particles; i.e., NaC's contain molecules not only DNA, but "guests" such as Chi, DAU molecules and copper ions. Hence, $\mathrm{NaC}$ 's of a new type can be designed.

Obviously, design of a specific spatial model of nanobridges between neighboring molecules in (DNA-Chi) complexes in the CLCD structure, and therefore, design of the spatial model of this $\mathrm{NaC}$, requires additional theoretical and experimental investigations.

\section{CONCLUSIONS}

In summary, we should note that the $\mathrm{NaC}$ 's formed on the basis of ds NA's, independent of the way of their formation, can find practical application in different fields of research and technology.

i) Nanoconstructions with the high DNA and "guest" concentration may be used as "carriers" for genetic material or as a "reservoir" for various biologically active compounds embedded in the composition of these structures. (The fields of application are medicine and biotechnology).

ii) Nanoconstructions based on particles of CLCD's of ds DNA (RNA) or (DNA-Chi) complexes can be used as sensing units for optical biosensors, which make it possible to determine the presence of relevant biologically active or chemical compounds in physiological liquids. (The fields of application are medicine, ecology, and biotechnology).

iii) Nanoconstructions with controlled physicochemical properties immobilized in synthetic polymeric films (hydrohels) can be used in technique (for example, as molecular sieves or optical filters). (The fields of application are optics and electronics).

The data reported here show that ds NA molecules are an important polyfunctional object for nanobiotechnology. Deliberate and controlled variation in the properties of these molecules provides wide possibilities for formation of nanobiostructures with unique properties.

\section{ACKNOWLEDGEMENT}

This research was supported in part by the grant from the Russian Foundation for Basic Research (project No 06-0408073).

\section{ABBREVIATIONS}

$$
\begin{array}{ll}
\mathrm{ds} & =\text { Double-stranded } \\
\mathrm{NA} & =\text { Nucleic acid }
\end{array}
$$




$$
\begin{array}{ll}
\mathrm{NaC} & =\text { Nanoconstruction } \\
\mathrm{NaC} ' s & =\text { Nanoconstructions } \\
\mathrm{LC} & =\text { Liquid-crystalline } \\
\mathrm{LCD} & =\text { Liquid-crystalline dispersion } \\
\mathrm{CLCD} & =\text { Cholesteric liquid-crystalline dispersion } \\
\mathrm{LCD} ' \mathrm{~s} & =\text { Liquid-crystalline dispersions } \\
\mathrm{CD} & =\text { Circular dichroism }
\end{array}
$$

\section{REFERENCES}

[1] Yevdokimov, Y.M.; Sitchev, V.V. Technol. Liv. Sys., 2007, 4, 3.

[2] Yevdokimov, Y.M.; Skuridin S.G.; Nechipurenko Y.D., Zakharov, M. A.; Salyanov, V.I.; Kurnosov A.A.; et al. Int. J. Biol. Macromol., 2005, 36, 103

[3] Feldkamp, U.; Niemeyer, C.M. Angew.Chem.Int. Ed., 2006, 45, 1856.

[4] Csaki, A.; Maubach, G.; Born, D.; Reichert J.; Fritzshe W. Single Mol., 2002, 3, 275.

[5] Hu, J,; Zhang, Y.; Gao, H.;Li, H.; Hartman, U. Nano Lett., 2002, 2, 55 .

[6] Seeman, N.C. Sci. Am., 2004, 6, 34.

[7] Fortina, P.; Kricka, L. K.; Surrey, S.; Grodzinski, P. Trends Biotechnol., 2005, 23, 168.

[8] Yevdokimov, Y.M.; Zakharov, M.A.; Skuridin, S.G. Herald Russ. Acad. Sci., 2006, 76, 5.

[9] La Bean, T.H.; Li H. Nanotoday, 2007, 2, 26

[10] Niemeyer, C.H. Nanotoday, 2007, 2, 42.

[11] Williams, K.A.; Veenhuizen, P.T.M.; de la Torre, B.G.; Eritja, R.; Dekker, C. Nature, 2002, 420, 761.

[12] Star, A.; Tu, E.; Niemann, J.; Gabriel, J.C.; Joiner, C.S.; Valcke, C. Proc. Natl. Acad. Sci., USA, 2006, 103, 921.

[13] Tang, X.; Bansaruntip, S.; Nakayama, N.; Yenilmez, E.; Chang, YL.; Wang, Q. Nano Lett., 2006, 6, 1632.

[14] Seeman, N.C. J. Theor. Biol., 1982, 99, 237.
[15] Chen, J.; Seeman, N.C. Nature, 1991, 350. 631.

[16] Shin, J.; Bergstrom, D. E. Angew. Chem. Int. Ed., 1997, 36, 111.

[17] Niemeyer, C. M.; Takeshi, S.; Smith, C. L. Nucleic Acids Res., 1994, 22, 5530 .

[18] Seeman, N.C. Sci. Amer., 2004, 6, 64

[19] Mirkin, C. A.; Mucic, R. C.; Storhoff, L. J. Nature, 1996, 382, 607.

[20] Mirkin, C.A.; Letsinger, R. L.; Mucic, R.C.; Storhoff, J.J. Nature, 1996, 382, 607.

[21] Alivisatos, A. P.; Johnsson, K. P.; Peng, X.; Wilson T.E.; Loweth C.J.; Bruchez M.P.; et al. Nature, 1996, 382, 609.

[22] Katz, E.; Willner, I. Angew. Chem. Int. Ed., 2004, 43, 6042

[23] Di Mauro, E.; Hollenberg, C. P. Adv. Mater., 1993, 5, 384.

[24] Onsager, L. Ann. N.Y. Acad. Sci., 1949, 51, 627.

[25] Livolant, F. Physica A, 1991, 176, 117.

[26] Livolant, F.; Leforestier, A. Prog. Polym.Sci., 1996, 21, 1115.

[27] Yevdokimov, Y.M.; Skuridin, S.G.; Salyanov, V.I. Liq.Cryst., 1988, 3, 1443.

[28] Yevdokimov, Y.M.; Skuridin S.G.; Lortkipanidze, G.B. Liq.Cryst., 1992, 12, 1 .

[29] Yevdokimov, Y.M. Herald Russ. Acad. Sci.,2006, 76, 5.

[30] Belyakov, V.A; Orlov, V.P.; Semenov, S.V.; Skuridin, S.G.; Lortkipanidze, G.B.; Chernukha, B.A.; et al. Liq. Cryst., 1996, 20, 777.

[31] Raspaud, E.; Olivera, de la Cruz M.; Sikorav, J. Biophys. J., 1998 74, 381 .

[32] Coble, H.D.; Holzclaw, H.F. J. Inorg. Nucl. Chem., 1974, 36, 1049. [33] Doskocil, J.; Fric, I. FEBS Lett., 1973, 37, 55.

[34] Nikiforov, V.I.; Kuznetsov, V.D.; Nechipurenko, Y.D. JEPT Lett., $\mathbf{2 0 0 5}, 81,327$.

[35] Golo V.L.; Kats, E.I., KIkot, I.P. JEPT Lett., 2006, 84, 334.

[36] Yevdokimov, Y.M.; Zakharov, M.A.; Salyanov, V.I. Crystallography Rep., 2006, 51, 1016.

[37] Yevdokimov, Y.M.; Salyanov, V.I. Liq. Cryst., 2003, 30, 1057.

[38] Schlich, S. Macromolecules, 1986, 19, 192.

[39] Domard, A. Int. J. Biol Macromol., 1987, 9, 98.

[40] Monteiro, O.A.C.; Arnoldi, C. J. Coll. Interface Sci., 1999, 212, 212.

[41] Inoue, K.; Baba, Y.; Yoshizuka, K. Bull. Chem. Soc. Japan, 1993, 66, 2915. 